\title{
Development of the $\mathrm{HOOS}_{\text {global }}$ patient-reported outcome measurement platform to assess patient-reported outcomes in hip preservation patients
}

\author{
Cale A. Jacobs, $\mathrm{PhD}^{1}$, Michael R. Peabody, $\mathrm{PhD}^{2}$, Stephen T. Duncan, MD ${ }^{1}$, Ryan D. \\ Muchow, MD ${ }^{1}$, Ryan M. Nunley, MD³ ${ }^{3}$ ANCHOR group ${ }^{*}$, John C. Clohisy, MD ${ }^{3}$ \\ ${ }^{1}$ Deparment of Orthopedic Surgery \& Sports Medicine, University of Kentucky, Lexington, KY \\ ${ }^{2}$ American Board of Family Medicine, Lexignton, $\mathrm{KY}$ \\ ${ }^{3}$ Department of Orthopedic Surgery, School of Medicine, Washington University, St. Louis, MO
}

\section{Abstract}

Background: The creation of a single patient-reported outcome (PRO) platform validated across hip preservation, osteoarthritis (OA), and total hip arthroplasty (THA) populations may reduce barriers and streamline routine collection of PROs in clinical practice. As such, the purpose of this study was to determine if augmenting the Hip dysfunction and Osteoarthritis Outcome Score, Joint Replacement (HOOS,JR) with addistional HOOS questions would result in a PRO platform that could be used across a wider spectrum of hip patient populations.

Hypothesis: The HOOS,JR would demonstrate a notable ceiling effect, but that augmenting the HOOS,JR with additional HOOS questions, a responsive PRO platform could be created.

Study Design: Case series. Level of Evidence: Diagnostic, Level II.

Methods: Using pre- and postoperative responses to the HOOS questionnaires from a sample of 304 peri-acetabular osteotomy patients, additional items were identified to augment the HOOS,JR. Psychometric properties of newly-created PRO tool ( $\left.\mathrm{HOOS}_{\text {global }}\right)$ were then compared to the HOOS,JR and other PRO instruments developed for hip OA and/or THA patients.

Results: By augmenting the HOOS,JR with two additional questions, the $\mathrm{HOOS}_{\text {global }}$ was more responsive than all other included PRO tools and had significantly fewer maximum postoperative scores than the HOOS,JR ( $\mathrm{p}<0.0001)$, HOOS-PS ( $\mathrm{p}<0.0001)$, WOMAC ( $\mathrm{p}=0.02)$, UCLA $(\mathrm{p}=0.0002)$, and $\mathrm{mHHS}$ scores $(\mathrm{p}=0.04)$. The postoperative threshold $\mathrm{HOOS}_{\text {global }}$ score associated with patients achieving an acceptable symptom state was 62.5 points.

Conclusion: The $\mathrm{HOOS}_{\text {global }}$ offers a valid and responsive PRO tool following PAO and may potentially provide the orthopedic community a PRO platform to be used across hip-related subspecialties. For patients undergoing peri-acetabular osteotomy, a postoperative $\mathrm{HOOS}_{\text {global }}$ score $\geq 62.5$ was associated with patients achieving an acceptable symptom state.

\footnotetext{
*ANCHOR Study Group Members who contributed to this article

Paul E. Beaulé, MD, FRCSC, Young-Jo Kim, MD, PhD, Michael B. Millis, MD, David A. Podeszwa, MD, Perry L. Schoenecker, MD, Rafael J. Sierra, MD, Ernest L. Sink, MD, Daniel J. Sucato, MD, Robert T. Trousdale, MD, and Ira Zaltz, MD

This study was performed at the University of Kentucky
} 


\section{Keywords}

hip; outcome; periacetabular osteotomy; responsiveness; patient-acceptable symptom state

\section{Introduction:}

The Hip dysfunction and Osteoarthritis Outcome Score (HOOS) is a 40-item patientreported outcome (PRO) tool that has provided invaluable information related to subjective pain and function for hip osteoarthritis (OA) and arthroplasty patients. ${ }^{22}$ However, while the full version of the HOOS allows for the specific domains of Pain, Symptoms, Activities of Daily Living (ADL), Sports and Recreation (Sport), and Quality of Life (QOL), the length of the tool has been a barrier to incorporating routine use into clinical practice. With the impending mandatory physician quality reporting for arthroplasty surgeons, Lyman et al. recently validated the HOOS Joint Replacement, or HOOS, JR. ${ }^{15}$ Consisting of six items from the full HOOS, the HOOS, JR is an efficient and responsive tool to that provides clinicians with a global score to assess postoperative hip arthroplasty outcomes. ${ }^{15}$ However, since the HOOS,JR was developed using a cohort of hip arthroplasty patients with an average age of 64 years, it is unlikely that the HOOS,JR would demonstrate similar psychometric properties if administered to younger, more active hip preservation patients.

There are clear advantages of having an efficient, valid and responsive global PRO measure, as meaningful clinical data can be generated with less burden to the patient, surgeon, and clinical staff. Furthermore, efficient global PRO scores may be more easily incorporated into the patient's electronic health record. Despite these advantages, to date there is no single PRO tool validated for use across hip-related subspecialties. PRO tools originally developed for the total hip arthroplasty (THA) community such as the Harris Hip Score have demonstrated large ceiling effects when administered to hip preservation patients. On the contrary, PRO tools such as the International Hip Outcome Tool (iHOT) and Hip Outcome Score (HOS) have been validated in the hip preservation population but not in the OA or THA population. ${ }^{16,18}$ The creation of a single PRO platform validated across hip preservation, OA, and THA populations may reduce barriers and streamline routine collection of PROs in clinical practice. As such, the purpose of this study was to develop a PRO platform by augmenting the HOOS, JR with additional questions from the full version of the HOOS, and to assess the validity and responsiveness of this tool in a hip preservation patient population. We hypothesized that when administered to a hip preservation patient

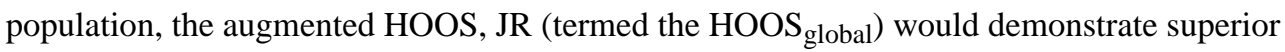
psychometric properties when compared to the HOOS, JR and other PRO tools that were originally developed for the OA and/or THA communities.

\section{Methods:}

\section{Patients}

From an IRB-approved, multicenter prospective cohort (Academic Network of Conservational Hip Outcomes Research (ANCHOR), Washington University IRB protocol \#201107167), we identified 304 consecutive peri-acetabular osteotomy (PAO) patients (283 
females, 61 males, age $=24.7 \pm 9.4$ years) with minimum 1-year follow-up and complete pre- and postoperative PRO data. All patients underwent PAO for hip dysplasia, with a subset having concomitant procedures to address cam $(n=16)$, pincer $(n=2)$, or combined deformity $(n=3)$. We included all PAO patients that had consented to participate in the study protocol, and patients were not excluded based on age, sex, race, or the presence of concomitant injury. In addition to the HOOS, patients routinely completed UCLA Activity Scale $^{32}$ and modified Harris Hip Score (mHHS) ${ }^{25}$ questionnaires both prior to and following surgery. From the full version of the HOOS, the HOOS, JR, ${ }^{15}$ HOOS-Physical Function Shortform (HOOS-PS), ${ }^{6}$ and Western Ontario and MacMaster Universities Arthritis Index $(\text { WOMAC })^{3}$ were calculated.

\section{Item selection}

To develop the $\mathrm{HOOS}_{\text {global }}$, we first identified individual items from the HOOS that were not included in the HOOS, JR for which a percentage of patients reported persistent postoperative difficulty. Items with $<33 \%$ of patients with postoperative responses of "none" were selected for possible inclusion in the $\mathrm{HOOS}_{\text {global }}$ meaning that at least two-thirds of patients continue to report pain or difficulty with that item at a minimum of one year after PAO. This was done to ensure the face and content validity of the $\mathrm{HOOS}_{\text {global }}$. We included only those items for possible inclusion for which a relatively high percentage of patients reported continued pain or difficulty one year after PAO. Items that relatively few patients reported pain or difficulty would not discriminate between patients with a poor or good outcome and by eliminating such items, the face and content validity of the $\mathrm{HOOS}_{\text {global }}$ would be maintained. Once potential additional items were identified, we applied a Rasch measurement model to the stacked dataset using both the HOOS, JR and HOOS global $_{\text {. }}$ 24, 28, 29 Because each item has its own distinct set of category descriptors, the analysis was conducted using the Rasch Partial Credit model available in Winsteps Rasch Measurement Software version 3.81.0 (Winsteps, Beaverton, OR). ${ }^{17,}{ }^{30}$ Details regarding the methods used in the Rasch analysis, item inclusion and exclusion, and comparative results of the HOOS,JR and $\mathrm{HOOS}_{\text {global }}$ can be found in the accompanying electronic Supplemental files.

We then calculated the $\mathrm{HOOS}_{\text {global }}$ score associated with a Patient-Acceptable Symptom State (PASS). The PASS represents the postoperative threshold value of a given PRO instrument that is associated with the majority of patients with postoperative scores above the PASS threshold are satisfied with their surgical procedure. ${ }^{9}$ To do so, we employed methods similar to those recently used to calculate the PASS for the International Knee Documentation Committee (IKDC) subjective knee form and Knee injury and Osteoarthritis Outcome Score (KOOS) after anterior cruciate ligament reconstruction. ${ }^{19}$ At the most recent postoperative follow-up visit, patients were asked if they were satisfied with their hip surgery with the options of either "Yes" or "No." We then utilized a Receiver Operator Characteristic (ROC) curve to determine if a PASS threshold for the HOOS $_{\text {global }}$ could be identified. The PASS threshold was determined by calculating the Youden index, ${ }^{31}$ which is product of the sensitivity and specificity for a given outcome score, and the PASS threshold was the combination of sensitivity and specificity with the greatest Youden index. 


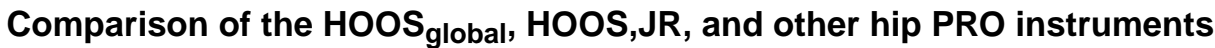

The responsiveness and the presence of floor and ceiling effects with the $\mathrm{HOOS}_{\text {global }}$ in comparison to the HOOS, JR and other PRO tools were then evaluated using the previously described methods. ${ }^{10}$ The responsiveness of the $\mathrm{HOOS}_{\text {global }}$ was also assessed by determining if the $\mathrm{HOOS}_{\text {global }}$ was able to detect expected differences between patients' preand postoperative scores. Responsiveness has been defined as a PRO tool's ability to detect meaningful or clinically important changes. ${ }^{11}$ Responsiveness was assessed using three commonly used methods: effect size (ES), standardized response mean (SRM), and relative efficiency (RE).${ }^{10,12,23} \mathrm{ES}$ is the mean pre- to postoperative change divided by the standard deviation of the preoperative scores, and ES $>0.8$ are considered large. ${ }^{5,}{ }^{10}$ SRM is the preto postoperative change divided by the standard deviation of the change scores for that particular PRO tool, ${ }^{11}, 12$ and SRM is interpreted similarly to ES, with values $>0.8$ considered large. The RE represents a ratio of the pre- to postoperative t-statistics of the HOOS $_{\text {global }}$ and each of the other PRO tools $\left[\mathrm{RE}=\left(\mathrm{t}_{\mathrm{KOOSglobal}} / \mathrm{t}_{\text {other PROs }}\right)^{2}\right] .{ }^{11,12} \mathrm{RE}$ values $>1$ are indicative of the $\mathrm{HOOS}_{\text {global }}$ being more responsive than the other PRO tools. The presence of a floor or ceiling effect was defined using the threshold of $15 \%$ of patients reporting either the worst possible or best possible scores, respectively. ${ }^{13}$ A ceiling effect is present when more than $15 \%$ of the sampled patients report the best possible score, and conversely, a floor effect is present when more than $15 \%$ of the sampled patients demonstrate the worst possible score. Neither floor and ceiling effects are ideal as both are indicative of a scoring system that can either not discriminate between an extremely poor result and a poor result (floor effect) or between an excellent and good result (ceiling effect). We have previously observed marked postoperative ceiling effects with OA and/or THAbased PRO tools in this patient population, and one-tailed chi-square tests were used to determine if the prevalence of maximum possible $\mathrm{HOOS}_{\text {global }}$ postoperative scores differed from the other PRO tools included in this study (HOOS, JR, HOOS-PS, WOMAC, UCLA, and $\mathrm{mHHS}$ ).

\section{Results:}

Based on the item selection methods and Rasch anslysis results, two additional items were identified from the full version of the HOOS that met the criteria for inclusion in the

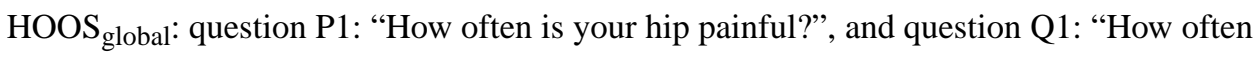
are you aware of your hip?" The raw HOOS $_{\text {global }}$ score was then determined by summing the responses to the original six HOOS, JR items and questions P1 and Q1. The scaled HOOS $_{\text {global }}$ score was determined using the logit conversion table and detailed results of the Rasch analyses, $\mathrm{HOOS}_{\text {global }}$ questionnaire, and scoring instructions can be found in the Supplemental Files available on the journal's website.

At mean follow-up of $4.0 \pm 1.5$ years, $\mathrm{HOOS}_{\text {global }}$ and all other PROs significantly improved from the preoperative visit (Tables 1,2). While all PRO tools including the $\mathrm{HOOS}_{\text {global }}$ exceeded the $15 \%$ threshold for ceiling effects $\left(\mathrm{HOOS}_{\text {global }}\right.$ had a $16 \%$ prevalence of maximum scores), the $\mathrm{HOOS}_{\text {global }}$ had significantly fewer maximum postoperative scores than the HOOS, JR ( $\mathrm{p}<0.0001)$, HOOS-PS ( $\mathrm{p}<0.0001)$, WOMAC $(\mathrm{p}=0.02)$, UCLA ( $\mathrm{p}=$ $0.0002)$, and mHHS scores $(\mathrm{p}=0.04$, Table 1$)$. A subset of 184 patients completed the 
postoperative satisfaction question, with 173 (94\%) reported being satisfied with their procedure and 11 were not satisfied (6\%). The PASS threshold score for $\mathrm{HOOS}_{\text {global }}$ was 62.5 (ROC curve Area Under Curve (AUC) $=0.81$ [95\% CI: 0.66, 0.96], $\mathrm{p}=0.001$ ), with sensitivity $=0.86$ and specificity $=0.73$ (Figure 1 ).

\section{Discussion:}

The purpose of this study was to develop a PRO platform by augmenting the HOOS, JR with additional questions from the full version of the HOOS, and to compare the validity and responsiveness of this tool to other PRO tools in a hip preservation patient population. The results by and large supported our hypothesis that the $\mathrm{HOOS}_{\text {global }}$ would demonstrate superior psychometric properties when compared to the HOOS, JR and other PRO tools that were originally developed for the OA and/or THA communities. By adding two additional items related to how often the patient has pain in the involved hip and how often the patient is aware of the hip significantly lessened the prevalence of patients with perfect postoperative scores. The HOOS, JR provides surgeons with a valid and efficient method to quantify postoperative outcomes following hip arthroplasty ${ }^{15}$; however, the HOOS, JR does not appear to be appropriate for use with hip preservation patient populations who have undergone periacetabular osteotomy surgery as a large ceilings effect was noted. This finding was not unique to the HOOS, JR as large ceiling effects were also present for the HOOS-PS, WOMAC, UCLA, mHHS and the five HOOS subscales after PAO. Not only did the $\mathrm{HOOS}_{\text {global }}$ alleviate some of the psychometric limitations of other OA-based PRO instruments, interpretation of $\mathrm{HOOS}_{\text {global }}$ results can be put in clinical context with postoperative scores $\geq 62.5$ were associated with patients achieving an acceptable symptom state.

These tools were initially created and validated largely in hip OA and THA patients ${ }^{1,3,7,22}$ and have proven to be effective measurement options for their target patient populations. Our results are similar to other previous reports that have also questioned the use of OA-related PRO instruments in populations of hip preservation patients. In a systematic review, Lodhia et al. reported notable ceiling effects with the WOMAC when used with femoroacetabular impingement and labral pathology. ${ }^{14}$ Similarly, Aprato et al. reported that $14 \%$ of patients with good to excellent modified Harris Hip Scores one year after hip arthroscopy were dissatisfied with their outcome, ${ }^{2}$ which could have been the result of a ceiling effect in this younger, more active patient population.

The goal of this work was not to supplant the HOOS, JR but rather to build upon it to reduce barriers to routine PRO collection in the clinical setting. The principal advantage of using the $\mathrm{HOOS}_{\text {global }}$ as a PRO platform is the versatility to be used across a variety of hip patient populations, thus reducing some of the burden to the orthopedic practice associated with routine PRO collection. The eight $\mathrm{HOOS}_{\text {global }}$ questions can be scored differently for different patient subsets. All eight questions can be used to generate a $\mathrm{HOOS}_{\text {global }}$ for hip preservation patients while the original six HOOS, JR questions can be scored for late stage OA and/or THA patients. Unlike the current available methods of using multiple PRO tools for hip preservation and arthroplasty subsets of patients, front office staff would not need to differentiate which hip PRO questionnaire to give an individual patient. For example, the 
front office staff for a clinician that normally sees both preservation and OA patients during the course of a clinic day would not have to determine which PROM tool to give each patient, but rather, could use the HOOS $_{\text {global }}$ for adult hip patients. Similarly, being able to use the $\mathrm{HOOS}_{\text {global }}$ could alleviate the complexities of collecting hip-related PROs in multisubspecialty practices. Perhaps most importantly, the eight questions of the $\mathrm{HOOS}_{\text {global }}$ take less than five minutes to complete, thereby minimizing the burden to the patient as well as reducing the likelihood that $\mathrm{PRO}$ collection will dramatically alter patient flow during the course of an average clinic day.

While the HOOS global may offer a single platform to reduce barriers to routine clinical PRO collection, we are not suggesting that this tool is a "silver bullet." The HOOS significantly reduced the prevalence of perfect postoperative scores when compared to OA/THA PRO tools, the $16 \%$ prevalence of perfect scores was above the $15 \%$ ceiling effect threshold. As such, future studies are necessary to determine if the HOOS $_{\text {global }}$ is an acceptable alternative to hip preservation-specific instruments, especially in athletic hip arthroscopy patients. Recommended PRO tools are available through the AAOS website; however, while the HOOS and HOOS, JR have been recommended for use with hip OA patients, ${ }^{26}$ the AAOS has not given guidance as to what tool(s) are best suited for the assessment of patients prior to and following hip preservation procedures. Multiple tools exist, including the International Hip Outcome Tool (iHOT), ${ }^{18}$ the Hip Outcome Score (HOS),${ }^{20}$ Nonarthritic Hip Score (NAHS), ${ }^{4}$ the Hip Sports Activity Scale (HSAS), ${ }^{21}$ and the Copenhagen Hip and Groin Outcome Score (HAGOS), ${ }^{27}$ Core Outcome Measures Index Hip (COMI-Hip), ${ }^{8}$ among others. While these have been utilized in the hip preservation literature, there is limited head-to-head comparative information related to the clinimetric and psychometric properties of these instruments. Also, it remains unclear if these hip preservation-specific tools could be utilized as patients transition from hip preservation to OA to arthroplasty, as they have not been validated in the OA or arthroplasty populations.

This study was not without limitation. First, an a priori power analysis was not performed as part of this study. Rather, we used liberal inclusion and exclusion criteria to ensure that the development of the HOOSglobal could be generalized to the broad PAO patient population. There is potential for a selection bias in that the study was performed with patients that elected to participate in a research study; however, because of the liberal inclusion/exclusion criteria there was little opportunity for selection bias on behalf of the researchers. Second, hip preservation-specific PRO instruments were not included as part of this multicenter prospective cohort. As such, we were unable to directly compare the responsiveness between OA- and hip preservation-specific PRO instruments. At the time that the multicenter registry was initiated, the psychometric properties of hip preservation-specific PRO tools had not yet been fully vetted, and since one of the primary goals of the registry is to evaluate long-term outcomes and the progression to OA, OA-based PRO tools were utilized. Third, the

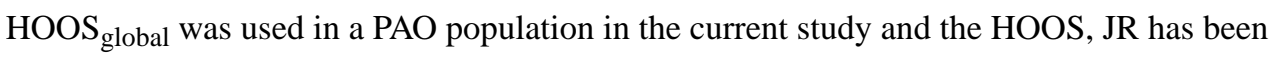
validated in the THA population. While these two patient populations represent the "anchors" on the continuum of post-traumatic osteoarthritis, the validity of the HOOS global platform has not been evaluated in either those with mild to moderate hip osteoarthritis or potentially younger and more active hip arthroscopy and/or FAI patient populations. Future 
studies are necessary to assess the test-retest reliability of the $\mathrm{HOOS}_{\mathrm{global}}$ and to determine if the current results are generalizable to additional patient groups.

In conclusion, the HOOS, JR and other OA-based PRO instruments demonstrated large ceiling effects in a hip preservation population following PAO. While we can conclude that OA-based PRO tools may not be the most appropriate method to quantify outcomes after hip preservation procedures due to the large ceiling effects that were observed, by including two additional HOOS questions, the $\mathrm{HOOS}_{\text {global }}$ offers a valid and responsive PRO tool following PAO and may potentially provide the orthopedic community a PRO platform to be used across hip-related subspecialties. Finally, postoperative $\operatorname{HOOS}_{\text {global }}$ scores $\geq 62.5$ were associated with patients achieving an acceptable symptom state.

\section{Supplementary Material}

Refer to Web version on PubMed Central for supplementary material.

\section{Acknowledgments}

Disclosure: This work was supported in part by Award UL1RR024992 from the National Center for Research Resources (J.C.C.). It was also supported in part by the Curing Hip Disease Fund (J.C.C.), the ANCHOR Research Fund, Smith \& Nephew (J.C.C.), and the NFL Charities (J.C.C.)

\section{References}

1. Amstutz HC, Thomas BJ, Jinnah R, Kim W, Grogan T, Yale C. Treatment of primary osteoarthritis of the hip: a comparison of total joint and surface replacement arthroplasty. J Bone Joint Surg (Am). 1984;66:228-241. [PubMed: 6693450]

2. Aprato A, Jayasekera N, Villar RN. Does the modified Harris hip score reflect patient satisfaction after hip arthroscopy? Am J Sports Med. 2012;40(11):2557-2560. [PubMed: 23024148]

3. Bellamy N, Buchanan WW, Goldsmith CH, Campbell J, Stitt LW. Validation study of WOMAC: A health status instrument for measuring clinically important patient relevant outcomes to antirheumatic drug therapies in patients with osteoarthritis of the hip or knee. J Rheumatol. 1988;15:1833-1840. [PubMed: 3068365]

4. Christensen CP, Althausen PL, Mittleman MA, Lee JA, McCarthy JC. The nonarthritic hip score: reliable and validated. Clin Orthop Related Res. 2003;406:75-83.

5. Cohen J Statistical power analysis for the behavioral sciences. Vol 2nd Hillsdale, NJ: Lawrence Earlbaum Associates; 1988.

6. Davis AM, Perruccio AV, Canizares M, et al. The development of a short measure of physical function for hip OA HOOS-Physical Function Shortform (HOOS-PS): an OARSI/OMERACT initiative. Osteoarthritis Cartilage. 2008;16(5):551-559. [PubMed: 18296074]

7. Harris WH. Traumatic arthritis of the hip after dislocation and acetabular fractures: treatment by mold arthroplasty. An end-result study using a new method of result evaluation. J Bone Joint Surg (Am). 1969;51:737-755. [PubMed: 5783851]

8. Impellizzeri FM, Mannion AF, Naal FD, Leunig M. Validation of the Core Outcome Measures Index in patients with femoroacetabular impingement. Arthroscopy. 2015;31(7):1238-1246. [PubMed: 25771423]

9. Kvien TK, Heiberg T, Hagen KB. Minimal clincally important improvement/difference (MCII/ MCID) and the patient acceptable symptom state (PASS): what do these concepts mean? Ann Rheum Dis. 2007;66(suppl 3):iii40-41.

10. Lam ETP, Lam CLK, Fong DYT, Huang WW. Is the SF-12 version 2 health survey a valid and equivalent substitute for the SF-36 version 2 health survey for the Chinese? J Eval Clin Pract. 2013;19:200-208. [PubMed: 22128754] 
11. Liang MH. Longitudinal construct validity: establishment of clinical meaning in patient evaluative instruments. Medical Care. 2000;38:1184-1190. [PubMed: 11186297]

12. Liang MH, Larson MG, Cullen KE, Schwartz JA. Comparative measurement efficiency and sensitivity of five health status instruments for arthritis research. Arthritis Rheum. 1985;28(5):542547. [PubMed: 4004963]

13. Lim CR, Harris K, Dawson J, Beard D, Fitzpatrick R, Price AJ. Floor and ceiling effects in the OHS: an analysis of the NHS PROMs data set. BMJ Open. 2015;5:e007765.

14. Lodhia P, Slobogean GP, Noonan VK, Gilbart MK. Patient-reported outcome instruments for femoroacetabular impingement and hip labral pathology: a systematic review of the clinimetric evidence. Arthroscopy. 2011;27(2):279-286. [PubMed: 21035994]

15. Lyman S, Lee Y-Y, Franklin PD, Li W, Mayman DJ, Padgett DE. Validation of the HOOS, JR: A short-form hip replacement survey. Clin Orthop Related Res. 2016;474:1472-1482.

16. Martin RL, Kelly BT, Philippon MJ. Evidence of validity for the hip outcome score. Arthroscopy. 2006;22(12):1304-1311. [PubMed: 17157729]

17. Masters GN. A Rasch Model for partial credit scoring. Psychometrika. 1982;47(2):149-174.

18. Mohtadi NG, Griffin DR, Pedersen ME, et al. The development and validation of a selfadministered quality-of-life outcome measure for young, active patients with symptomatic hip disease: the International Hip Outcome Tool (iHOT-33). Arthroscopy. 2012;28(5):595-605. [PubMed: 22542433]

19. Muller B, Yabroudi MA, Lynch A, et al. Defining thresholds for the patient acceptable symptom state for the IKDC subjective knee form and KOOS for patients who underwent ACL reconstruction. Am J Sports Med. 2016;44(11):2820-2826. [PubMed: 27474383]

20. Naal FD, Impellizzeri FM, Miozzari HH, Mannion AF, Leunig M. The German Hip Outcome Score: validation in patients undergoing surgical treatment for femoroacetabular impingement. Arthroscopy. 2011;27(3):339-345. [PubMed: 21071169]

21. Naal FD, Miozzari HH, Kelly BT, Magennis EM, Leunig M, Noetzli HP. The Hip Sports Activity Scale (HSAS) for patients with femoroacetabular impingement. Hip Int. 2013;23(2):204-211. [PubMed: 23543465]

22. Nilsdotter A-K, Lohmander LS, Klassbo M, Roos EM. Hip disability and osteoarthritis outcome score (HOOS)--validity and responsiveness in total hip replacement. BMC Musculoskeletal Disorders. 2003;4:10. [PubMed: 12777182]

23. Nilsdotter A-K, Roos EM, Westerlund JP, Roos HP, Lohmander LS. Comparative responsiveness of measures of pain and function after total hip replacement. Arthritis Care \& Research. 2001;45:258-262. [PubMed: 11409667]

24. Rasch G Probabilistic Models for Some Intelligence and Attainment Tests. Copenhagen, Denmark: Danish Institute for Educational Research; 1960.

25. Shamra S, Shah R, Draviraj KP, Bhamra MS. Use of telephone interviews to follow up patients after total hip replacement. J Telemedicine and Telecare. 2005;11:211-214.

26. Surgeons AAoO. Patient Reported Outcome Measures. Accessed 12/7/2016.

27. Thorborg K, Holmich P, Christensen R, Petersen J, Roos EM. The Copenhagen Hip and Groin Outcome Score (HAGOS): development and validation according to the COSMIN checklist. Br J Sports Med. 2011;45(6):478-491. [PubMed: 21478502]

28. Wright BD. Time 1 to Time 2 (Pre-test to Post-test) comparison: Racking and Stacking. Rasch Measurement Transactions. 1996;10(1):478.

29. Wright BD. Rack and Stack: Time 1 vs. Time 2 or Pre-Test vs. Post-Test. Rasch Measurement Transactions. 2003;17(1):905-906.

30. Wright BD, Masters GN. Rating Scale Analysis. Chicago, IL: MESA Press; 1982.

31. Youden WJ. Index for rating diagnostic tests. Cancer. 1950;3(1):32-35. [PubMed: 15405679]

32. Zahiri CA, Schmalzried TP, Szuszczewicz ES, Amstutz HC. Assessing activity in joint replacement patients. J Arthroplasty. 1998;13(8):890-895. [PubMed: 9880181] 


\section{What is known about the subject:}

The HOOS, JR offers an efficient method to evaluate postoperative outcomes following THA; however, the ability to utilize this PROM tool in younger, more active hip preservation patient population has not been determined.

What this study adds to the existing knowledge:

The HOOS, JR is not a valid or responsive tool to assess patient-reported outcomes following peri-acetabular osteotomy; however, an aggregate score combining HOOS, JR with the 2 additional HOOS questions, was valid and responsive in this patient population. 


\section{HOOSglobal HIP SURVEY}

INSTRUCTIONS: This survey asks for your view about your hip. This information will help us keep track of how you feel about your hip and how well you are able to do your usual activities.

Answer every question by ticking the appropriate box, only one box for each question. If you are uncertain about how to answer a question, please give the best answer you can.

Pain

1. How often is your hip painful?
Never
Monthly
Weekly
Daily
Always
$\square$

$\square$

$\square$

$\square$

$\square$

What amount of hip pain have you experienced the last week during the following activities?
2. Going up or down stairs
None Mild
$\square$
$\square$
Moderate
Severe
Extreme
3. Walking on an uneven surface
None
Mild
Moderate
Severe
$\square$
$\square$
$\square$
$\square$
$\square$
Extreme
$\square$

\section{Function, daily living}

The following questions concern your physical function. By this we mean your ability to move around and to look after yourself. For each of the following activities please indicate the degree of difficulty you have experienced in the last week due to your hip.
4. Rising from sitting
None
Mild
Moderate
Severe
Extreme
$\square$
$\square$
$\square$
$\square$
$\square$
5. Bending to the floor/pick up an object

None Mild Severe $\quad$ Extreme
$\square$
口
$\square$
$\square$
$\square$
6. Lying in bed (turning over, maintaining hip position)
None $\quad$ Mild $\quad$ Moderate $\quad$ Severe $\quad$ Extreme
$\square$
$\square$
$\square$
$\square$
$\square$
7. Sitting
None
Mild
Moderate
Severe
Extreme
$\square$
$\square$
$\square$

\section{Quality of Life}

8. How often are you aware of your hip problem?
Never
Monthly
Weekly
Daily
Constantly

口

$\square$

$\square$

Thank you very much for completing all the questions

in this questionnaire.

Figure 1.

HOOSglobal hip survey. 


\section{HOOSglobal SCORING INSTRUCTIONS}

The HOOSglobal was developed from the original versions of the Hip dysfunction and Osteoarthritis Outcome Score (HOOS) and HOOS, JR surveys and contains 8 items from the original surveys. Items are coded from 0 to 4 , none to extreme respectively.

HOOSglobal is scored by summing the raw response (range $0-32$ ) and then converting it to an interval score using the table provided below. The interval score ranges from 0 to 100 where 0 represents complete hip disability and 100 represents perfect hip health.

Table for converting raw summed scores to interval scores from 0 (complete hip disability) to 100 (perfect hip health)

\begin{tabular}{ccccc}
\hline $\begin{array}{c}\text { Raw summed } \\
\text { score (0-32) }\end{array}$ & $\begin{array}{c}\text { Interval score } \\
\text { (0 to 100 scale) }\end{array}$ & & $\begin{array}{c}\text { Raw summed } \\
\text { score (0-32) }\end{array}$ & $\begin{array}{c}\text { Interval score } \\
\text { (0 to 100 scale) }\end{array}$ \\
\hline 0 & 100.000 & & 17 & 49.009 \\
1 & 90.943 & & 18 & 47.161 \\
2 & 83.620 & & 19 & 45.269 \\
3 & 78.468 & & 20 & 43.321 \\
4 & 74.799 & & 21 & 41.297 \\
5 & 71.946 & 22 & 39.171 \\
6 & 69.530 & & 23 & 36.907 \\
7 & 67.360 & & 24 & 34.449 \\
8 & 65.341 & & 25 & 31.745 \\
9 & 63.419 & & 26 & 28.772 \\
10 & 61.561 & & 27 & 25.569 \\
11 & 59.745 & & 28 & 22.172 \\
12 & 57.954 & & 29 & 18.488 \\
13 & 56.177 & 30 & 14.189 \\
14 & 54.401 & & 32 & 8.280 \\
15 & 52.619 & & & 0.000 \\
16 & 50.825 & & 30 & \\
\hline
\end{tabular}

Figure 2.

HOOSglobal scoring instructions. 
ROC Curve

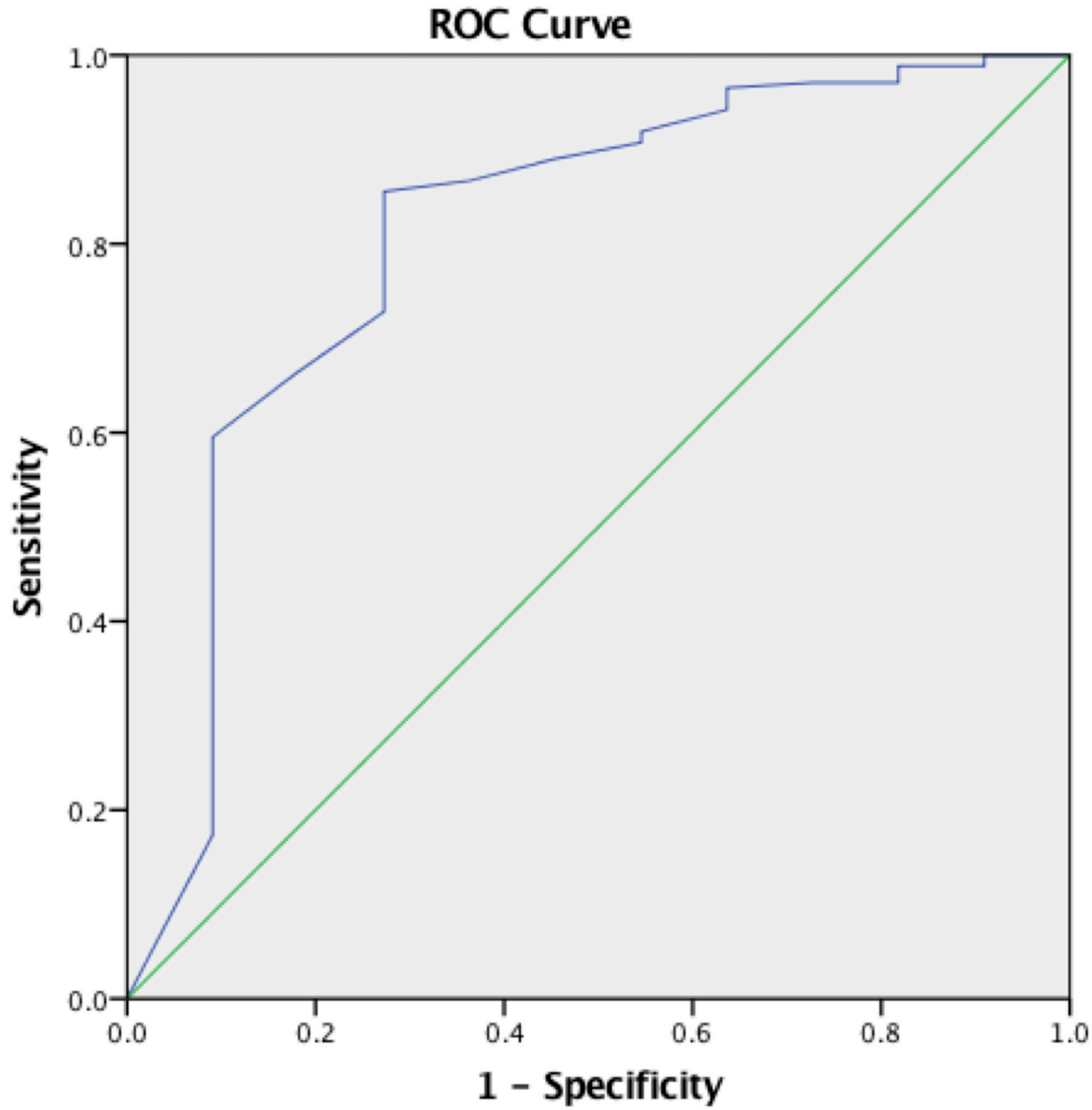

Diagonal segments are produced by ties.

Figure 3.

Receiver Operator Characteristic (ROC) curve used to determine the postoperative HOOS $_{\text {global }}$ score threshold associated with postoperative patient satisfaction 


\section{를}

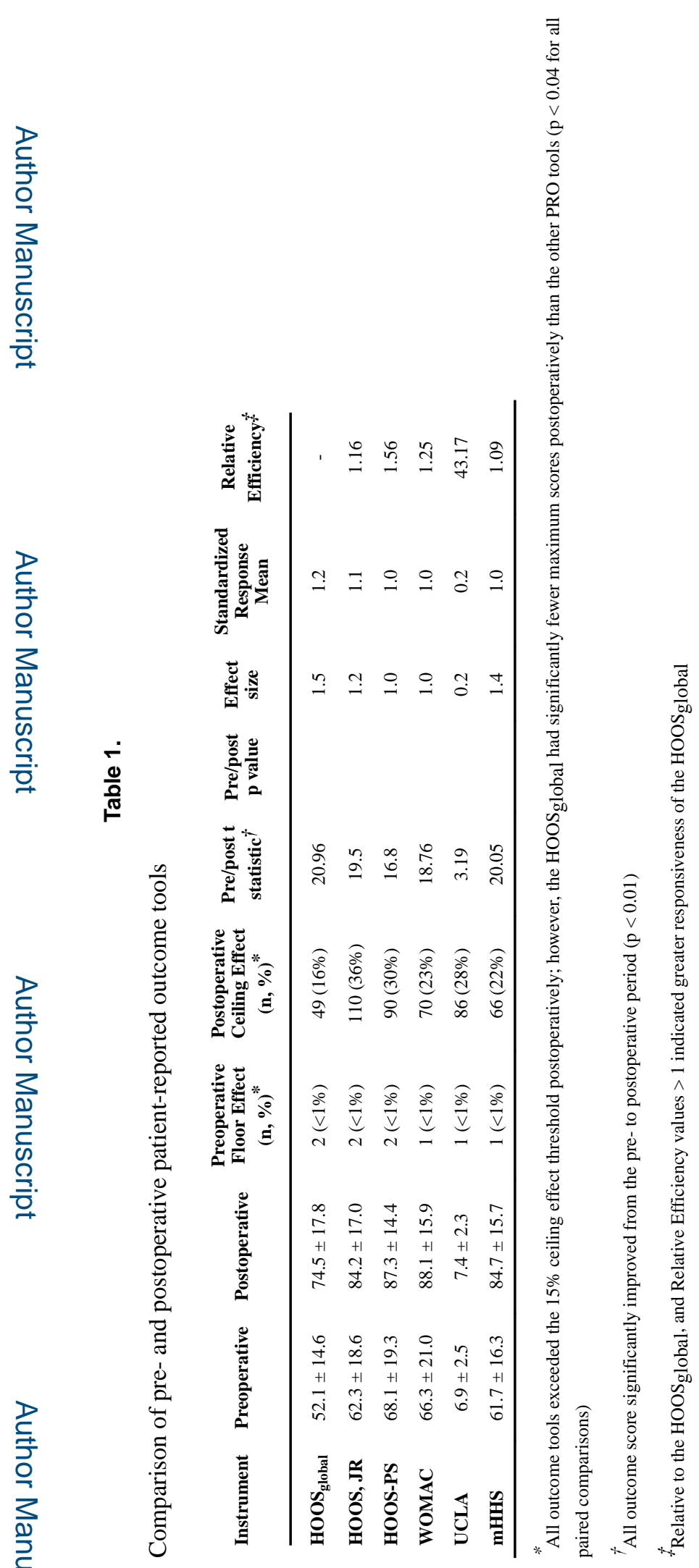




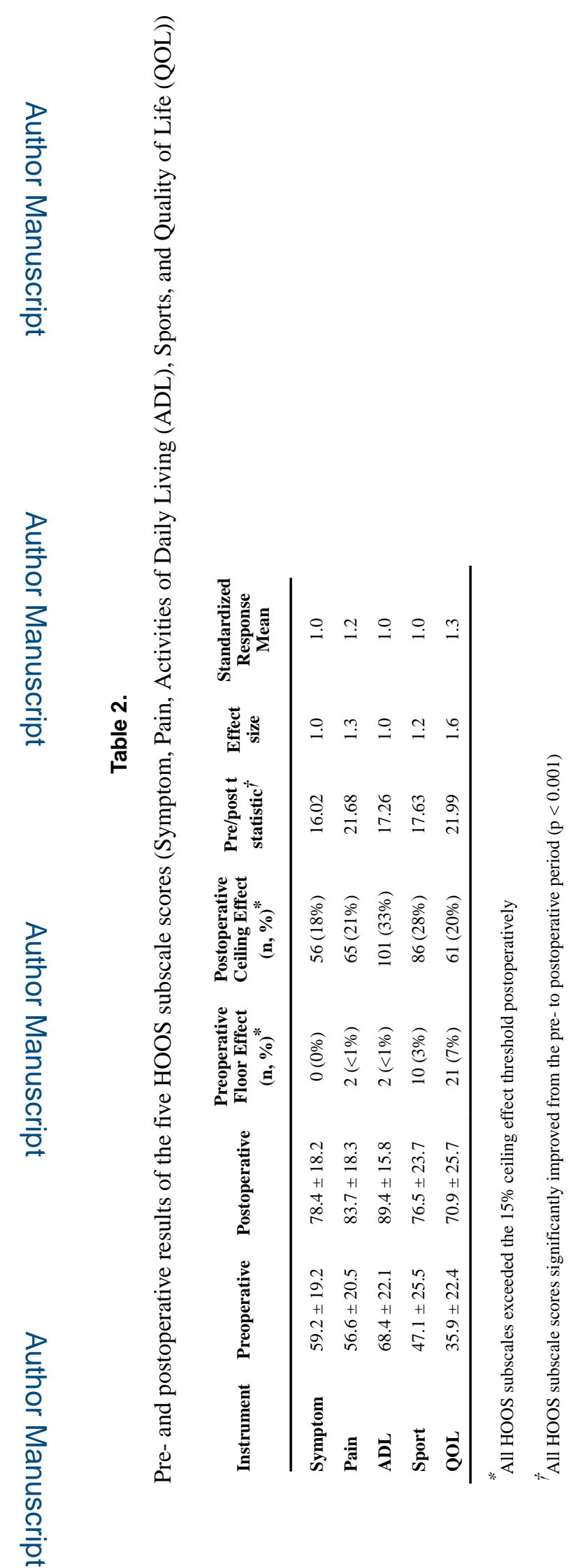

Am J Sports Med. Author manuscript; available in PMC 2020 January 05. 